Pacific Journal of Mathematics

INDEX FOR FACTORS GENERATED BY JONES' TWO SIDED
SEQUENCE OF PROJECTING 


\title{
INDEX FOR FACTORS GENERATED BY JONES' TWO SIDED SEQUENCE OF PROJECTIONS
}

\author{
MARIE Choda
}

Let $\left\{e_{i} ; i=0, \pm 1, \pm 2, \ldots\right\}$ be a family of projections with the property; (a) $e_{i} e_{\imath \pm 1} e_{1}=\lambda e_{l}$ for some $\lambda \leq 1$, (b) $e_{l} e_{J}=e_{j} e_{l}$ for $\mid i-$ $j \mid \geq 2$, (c) the von Neumann algebra $M$ generated by the family $\left\{e_{i} ; i=0, \pm 1, \pm 2, \ldots\right\}$ is a hyperfinite $\mathrm{II}_{1}$-factor with the trace $\mathrm{tr}$ and (d) $\operatorname{tr}\left(w e_{t}\right)=\lambda \operatorname{tr}(w)$ if $w$ is a word on 1 and $e_{j}(j \leq i-1)$. Let $N$ be a von Neumann algebra generated by $\left\{e_{l} ; i= \pm 1, \pm 2, \ldots\right\}$. Then $N$ is a subfactor of $M$. If $\lambda=(1 / 4) \sec ^{2}(\pi / m)$ for some integer $m(m \geq 3)$, then $N^{\prime} \cap M=\mathrm{C} 1$ and the index $[M: N]=(m / 4) \operatorname{cosec}^{2}(\pi / m)$.

1. Introduction. The index theory for finite factors was introduced by Jones in [3]. In that paper, the following sequence $\left\{e_{i} ; i=1,2, \ldots\right\}$ of projections plays an important role:

(a) $e_{i} e_{i \pm 1} e_{i}=\lambda e_{i}$ for some $\lambda \leq 1$,

(b) $e_{i} e_{j}=e_{j} e_{i}$ for $|i-j| \geq 2$,

(c) the von Neumann algebra $P$ generated by $\left\{e_{i} ; i=1,2, \ldots\right\}$ is a hyperfinite $\mathrm{II}_{1}$-factor,

(d) $\operatorname{tr}\left(w e_{i}\right)=\lambda \operatorname{tr}(w)$ if $w$ is a word on $1, e_{1}, e_{2}, \ldots, e_{i-1}$, where $\operatorname{tr}$ is the canonical trace of $P$ and 1 is the identity operator.

If $Q$ is the subfactor of $P$ generated by $\left\{e_{i} ; i=2,3, \ldots\right\}$, then the index $[P: Q]$ of $Q$ in $P$ is $1 / \lambda$. In the case $\lambda>1 / 4, Q$ has trivial relative commutant in $P$ and $[P: Q]=4 \cos ^{2}(\pi / m)$ for some $m=$ $3,4, \ldots$ Hence by his basic construction, we have the family $\left\{e_{i} ; i=\right.$ $\ldots,-2,-1,0-, 1,2, \ldots\}$ of projections with the properties $(\mathrm{a}),(\mathrm{b}),\left(\mathrm{c}^{\prime}\right)$ and $\left(\mathrm{d}^{\prime}\right)$;

$\left(\mathrm{c}^{\prime}\right)\left\{e_{i} ; i=0, \pm 1, \pm 2, \ldots\right\}$ generates a hyperfinite $\mathrm{II}_{1}$ factor $M$,

$\left(\mathrm{d}^{\prime}\right) \operatorname{tr}\left(w e_{i}\right)=\lambda \operatorname{tr}(w)$ for the trace $\operatorname{tr}$ of $M$ if $w$ is a word on 1 and $\left\{e_{j} ; j<i\right\}$ (cf. [5]).

We shall call this family $\left\{e_{i} ; i=0, \pm 1, \pm 2, \ldots\right\}$ the Jones' two sided sequence of projections for $\lambda$. The main purpose of this note is to show the following theorem.

Theorem. Let $\left\{e_{i} ; i=0, \pm 1, \pm 2, \ldots\right\}$ be the Jones' two sided sequence of projections for $\lambda=(1 / 4) \sec ^{2}(\pi / m)$ for some $m(m=3,4, \ldots)$. If $M($ resp. $N)$ is the von Neumann algebra generated by $\left\{e_{i} ; i=\right.$ $0, \pm 1, \pm 2, \ldots\}$ (resp. $\left\{e_{i} ; i= \pm 1, \pm 2, \ldots\right\}$ ), then $N$ is a subfactor of $M$ 
with the index

$$
[M: N]=(m / 4) \operatorname{cosec}^{2}(\pi / m),
$$

and the relative commutant of $N$ in $M$ is trivial, that is, $N^{\prime} \cap M=\mathbf{C} 1$.

The author learned from the referee that A. Ocneanu obtained the same formula independently. She would like to express her hearty thanks to the referee for many valuable comments.

2. Notations and preliminaries. Let $B$ be a subfactor of a $\mathrm{II}_{1}$-factor $A$. Then Jones defined in [3] the index $[A: B]$ of $B$ in $A$ using the coupling constants of $A$ and $B$ due to Murray and von Neumann ([4]) and he (and also, Pimsner-Popa in [5]) gives some methods to get the number $[A: B]$. In [6], Wenzl gets another method to compute $[A: B]$ in the case where those factors are $\sigma$-weak closures of the union of increasing sequences of finite dimensional algebras, which satisfy some good conditions.

In this note, we shall use the results in [6] to give a proof of Theorem.

(2.1) Let $A$ be a finite dimensional von Neumann algebra. Then $A$ is decomposed into a direct sum $\sum_{i=1}^{m} \oplus A_{i}$ of $a(i)$ by $a(i)$ matrix algebra $A_{i}$. The vector $a=(a(i))$ is called the dimension vector of $A$, following Wenzl [6]. Each trace $\phi$ on the algebra $A$ is determined by a column vector $w=(w(i))$ which satisfies $\phi(x)=\sum_{i=1}^{m} w(i) \operatorname{Tr}\left(x_{i}\right)$ for $x \in A$, where $x=\sum \oplus x_{i}\left(x_{i} \in A_{i}\right)$ and $\operatorname{Tr}$ is the usual nonnormalized trace on the matrix algebra. The row vector $w$ is called the weight vector of the trace $\phi$. Let $B$ be a von Neumann subalgebra of $A$ with direct summand $B=\sum_{i=1}^{n} \oplus B_{i}$ of $b(i)$ by $b(i)$ matrix algebras $B_{i}$. The inclusion of $B$ in $A$ is specified up to conjugacy by an $n$ by $m$ matrix [ $\left.g_{i, j}\right]$, where $g_{i, j}$ is the number of simple components of a simple $A_{j}$ module viewed as a $B_{i}$ module. The matrix $\left[g_{i, j}\right]$ is called the inclusion matrix of $B$ in $A$ which we denote by $[B \rightarrow A]$. Let $b=(b(i))$ be the dimension vector of $B$ and $v$ the weight vector of the restriction of $\phi$ of $B$, then

(e) $b[B \rightarrow A]=a$ and $[B \rightarrow A] w=v$.

(2.2) Let $\left\{e_{i} ; i=0, \pm 1, \pm 2, \ldots\right\}$ be Jones' two sided sequence of projections for $\lambda(\lambda \leq 1)$. A reduced word is a word on $e_{i}$ 's of minimal length for the rules (a), (b) and $e_{i}^{2} \leftrightarrow e_{i}$. As a trivial consequence of Jones' method in [3], we have that the von Neumann algebra $N$ generated by $\left\{e_{i} ; i= \pm 1, \pm 2, \ldots\right\}$ is a subfactor of the hyperfinite $\mathrm{II}_{1}$ factor $M$ generated by $\left\{e_{i} ; i=0, \pm 1, \pm 2, \ldots\right\}$. 
(2.3) The factor $M$ is the $\sigma$-weak closure of the union of the increasing sequence of the following von Neumann algebras $\left\{M_{k} ; k=\right.$ $1,2, \ldots\}$ :

$$
M_{1}=\mathrm{C} 1, \quad M_{2 m}=\left\{e_{j} ;|j| \leq m-1\right\}^{\prime \prime}, \quad M_{2 m+1}=\left\{M_{2 m}, e_{m}\right\}^{\prime \prime} .
$$

The subfactor $N$ of $M$ is generated by the following increasing sequence of $\left\{N_{k} ; k=1,2, \ldots\right\}$ :

$N_{1}=N_{2}=\mathbf{C}_{1}, \quad N_{2 m}=\left\{e_{j} ; 0 \neq|j| \leq m-1\right\}^{\prime \prime}, \quad N_{2 m+1}=\left\{N_{2 m}, e_{m}\right\}^{\prime \prime}$.

The algebras $M_{k}$ and $N_{k}$ are all finite dimensional ([3]). We denote by $a_{k}$ (resp. $b_{k}$ ) the dimension vector of $M_{k}$ (resp. $N_{k}$ ). In the case where $M_{k}$ is the direct sum of $d_{k}$ matrix algebras, we say $d_{k}$ is the length of the dimension vector $a_{k}$.

(2.4) Every $N_{k}$ is a subalgebra of $M_{k}$. Let $E(B)$ be the conditional expectation of $M$ onto the von Neumann subalgebra $B$ of $M$ conditioned by $\operatorname{tr}(x E(B)(y))=\operatorname{tr}(x y)$ for $x \in B$ and $y \in M$.

Lemma 1. $E\left(N_{k+1}\right) E\left(M_{k}\right)=E\left(N_{k}\right)$ and $E(N) E\left(M_{k}\right)=E\left(N_{k}\right)$ for all $k$.

Proof. Since $E\left(N_{k+1}\right) E\left(M_{k}\right)=E\left(N_{k}\right)$ if and only if $E\left(N_{k+1}\right) E\left(M_{K}\right)$ $=E\left(N_{k+1}\right) E\left(N_{k}\right) E\left(M_{k}\right)$, it is sufficient to prove that $\operatorname{tr}\left(y E\left(N_{k+1}\right)(x)\right)$ $=\operatorname{tr}\left(y E\left(N_{k}\right)(x)\right)$, for $x \in M_{k}, y \in N_{k+1}$. Every reduced word $y \in$ $N_{2 m+1}$ has a form $y=v w_{1} e_{m} w_{2}$, where $v$ is a reduced form on $\left\{e_{i} ; i=\right.$ $-m+1,-m+2, \ldots,-1\}$ and $w_{i}(i=1,2)$ is a reduced word on $\left\{e_{i} ; i=1,2, \ldots, m-1\right\}$. Let $w$ be a reduced word in $M_{2 m}$; then

$$
\begin{aligned}
\operatorname{tr}\left(y E\left(N_{2 m+1}\right)(w)\right) & =\operatorname{tr}(y w)=\lambda \operatorname{tr}\left(w_{2} w v w_{1}\right)=\lambda \operatorname{tr}\left(E\left(N_{2 m}\right)(w) v w_{1} w_{2}\right) \\
& =\operatorname{tr}\left(w_{2} E\left(N_{2 m}\right)(w) v w_{1} e_{m}\right)=\operatorname{tr}\left(y E\left(N_{2 m}\right)(w)\right) .
\end{aligned}
$$

Since each algebra is generated by reduced words, $E\left(N_{2 m+1}\right) E\left(M_{2 m}\right)$ $=E\left(N_{2 m}\right)$. Similarly $E\left(N_{2 m}\right) E\left(M_{2 m+1}\right)=E\left(N_{2 m-1}\right)$. Since $E\left(N_{k+1}\right) E\left(M_{k}\right)=E\left(N_{k+i}\right) E\left(M_{k+i-1}\right) E\left(M_{k}\right)=E\left(N_{k+i-1}\right) E\left(M_{k}\right)=$ $\cdots=E\left(M_{k}\right)$

$$
E(N) E\left(M_{k}\right)=E\left(M_{k}\right) \text { for all } k \text {. }
$$

(2.5) Let $\left(A_{k}\right)$ and $\left(B_{k}\right)$ be sequences of finite dimensional von Neumann algebras such that $B_{k} \subset A_{k}$ for all $k$. Following after [6], we write $\left(A_{k}\right)_{k} \supset\left(B_{k}\right)_{k}$ if $\left(A_{k}\right)_{k}$ (resp. $\left.\left(B_{k}\right)_{k}\right)$ generates a $\mathrm{II}_{1}$-factor $A$ (resp. a subfactor $B$ of $A$ ) and satisfies the property of Lemma 1. So, 
by $\left(\mathrm{c}^{\prime}\right),(2.2)$ and Lemma 2 , we have $\left(N_{k}\right)_{k} \subset\left(M_{k}\right)_{k}$. Such a sequence $\left(M_{k}\right)$ is said to be periodic with period $r$ if there is a number $m$ such that $\left[M_{n+r} \rightarrow M_{n+r+i}\right]=\left[M_{n} \rightarrow M_{n+l}\right]$ for $n \geq m(i=1,2, \ldots)$ and the matrix $\left[M_{n} \rightarrow M_{n+r}\right]$ is primitive for $n \geq m$. The sequences $\left(M_{k}\right)_{k} \supset\left(N_{k}\right)_{k}$ is periodic if both $\left(M_{k}\right)$ and $\left(N_{k}\right)$ are periodic with same period $r$ and $\left[N_{n+r} \rightarrow M_{n+r}\right]=\left[N_{n} \rightarrow M_{n}\right.$ ] for a large enough $n$ ([6]). In Section 6, we show the periodicity of $\left(N_{k}\right)_{k} \subset\left(M_{k}\right)_{k}$.

3. Bratteli diagram for $\left(M_{k}\right)_{k}$ and path maps. For convenience' sake, throughout we put

(3.1) for a positive integer $k, p=[k / 2]$ and $q=k-p$.

In this section, we shall get, for the sequence $\left(M_{k}\right)$ in (2.3), the components of the inclusion matrix $\left[M_{q} \rightarrow M_{k}\right.$ ], which we need to obtain the inclusion matrix $\left[N_{k} \rightarrow M_{k}\right]$. Let $A_{k}=\left\{1, e_{1}, \ldots, e_{k}\right\}^{\prime \prime}$. Then $M_{k}$ is $*$-isomorphic to $A_{k-1}$ for $k \geq 2$. On the other hand there is a unitary $u$ in $M_{2 m}$ which satisfies $u e_{i} u^{*}=e_{-i}$ and $u e_{-i} u^{*}=e_{i}$ for all $i=0,1, \ldots, m-1([3])$. Hence $\left[M_{k} \rightarrow M_{k+1}\right]=\left[A_{k-1} \rightarrow A_{k}\right]$ for all $k \geq 2$. It is clear that $\left[M_{1} \rightarrow M_{2}\right]$ is the 1 by 2 matrix [1,1]. In [3], Jones gets the Bratteli diagram ([1]) for the sequence $\left(A_{k}\right)$ and so we get the Bratteli diagram for $\left(M_{k}\right)$. The dimension vector $a_{k}$ of $M_{k}$, the length $d_{k}$ of $a_{k}$ and the weight vector $w_{k}$ of the restriction of $\operatorname{tr}$ on $M_{k}$ are as follows:

(3.2) If $\lambda \leq 1 / 4$, then

$$
\begin{aligned}
& d_{k}=p+1 \text {, } \\
& a_{k}(i)= \begin{cases}\left(\begin{array}{c}
k \\
p+1-i
\end{array}\right)-\left(\begin{array}{c}
k \\
p-i
\end{array}\right) & \text { if } i=1,2, \ldots, d_{k}-1, \\
1 & \text { if } i=d_{k},\end{cases} \\
& w_{k}(i)=\lambda^{p+1-i} P_{k-1-2 p+2 i}(\lambda),
\end{aligned}
$$

where $P_{j}$ is the polynomial defined in [3] by $P_{1}(x)=P_{2}(x)=1$ and $P_{n+1}(x)=P_{n}(x)-x P_{n-1}(x)$.

$$
\left[M_{k} \rightarrow M_{k+1}\right]=\left[\delta_{i, j}+\delta_{i+1, j}\right]_{i, j}, \quad \text { for Kronecker's } \delta_{l, j},
$$

where $i=1,2, \ldots,[(k+1) / 2]+1$ and

$$
j= \begin{cases}1,2, \ldots,[(k+1) / 2]+1 & \text { if } k \text { is even } \\ 1,2, \ldots,(k+3) / 2 & \text { if } k \text { is odd }\end{cases}
$$


(3.3) If $\lambda>1 / 4$, then $\lambda=(1 / 4) \sec ^{2}(\pi / n+2)$ for some $n=1,2, \ldots$ The Blatteri diagram for $M_{1} \subset M_{2} \subset \cdots \subset M_{n}$ has the same form as in the case of $\lambda \leq 1 / 4$ and the diagram for $M_{n+2 i-1} \subset M_{n+2 i}$ (resp. $\left.M_{n+2 i} \subset M_{n+2 i-1}\right)$ is the same as the one for $M_{n-1} \subset M_{n}$ (resp. the reverse form of one for $M_{n-1} \subset M_{n}$ ), for all $i=0,1,2, \ldots$.

Now we consider the Bratteli diagram for $\left(M_{k}\right)_{k}$ as a graph $\Lambda$, the set of vertices of which is the set of points where $a_{k}(i)(k=1,2, \ldots, i=$ $\left.1,2, \ldots, d_{k}\right)$ stand. We denote the vertex in $\Lambda$ corresponding to $a_{k}(i)$ by the same notation $a_{k}(i)$. We denote by $\left[a_{k}(i) \rightarrow a_{k+1}(j)\right]$ the edge from $a_{k}(i)$ to $a_{k+1}(j)$. A path on $\Lambda$ is a sequence $\xi=\left(\xi_{r}\right)$ of edges such that $\xi_{r}=\left[a_{k(r)}\left(i_{r}\right) \rightarrow a_{k(r)+1}\left(j_{r}\right)\right]$ for some $i_{r}, j_{r}$ and $k(r)$ such that $k(r+1)=k(r)+1$. The set of all paths in $\Lambda$ with the starting point $a_{k}(i)$ and the ending point $a_{r}(j)$ is called a polygon from the vertex $a_{k}(i)$ to the vertex $a_{r}(j)$ and denoted by $\left[a_{k}(i) \rightarrow a_{r}(j)\right]$. Also the set of all paths in $\Lambda$ with $a_{k}(i)$ as the starting point and for some $j a_{r}(j)$ as the ending point is called a path map from the vertex $a_{k}(i)$ to the floor $a_{r}$ and denoted by $\left[a_{k}(i) \rightarrow a_{r}\right]$. Let $\Xi_{m}$ be the set of paths on $\Lambda$ consisting of $m$ edges. For a $\xi$ in $\Xi_{1}$ and $y$ in $\Xi_{m}$ let $\xi \circ y=\{\xi \circ \eta ; \eta \in y\}$. Let $x \in \Xi_{m}$ be a polygon. If there are polygons $y$ and $z$ in $\Xi_{m-1}$ such that as sets of paths $x$ is either the union of $\xi \circ y$ and $\eta \circ z$ or the union of $y \circ \xi$ and $z \circ \eta$ for some $\xi$ and $\eta$ in $\Xi_{1}$, we say $x$ is the direct sum of $y$ and $z$ and we write $x=y \oplus z$ for $y=x \ominus z$.

REMARK 2. The $i$ th coordinate $a_{k}(i)$ of the dimension vector $a_{k}$ represents a cardinal number of different paths in the polygon $\left[a_{1}(1) \rightarrow\right.$ $a_{k}(i)$ ]. Below, we consider $a_{k}(i)$ as the polygon $\left[a_{1}(1) \rightarrow a_{k}(i)\right]$ and the dimension vector $a_{k}$ as the path map $\left[a_{1}(1) \rightarrow a_{k}\right]$. Also, for path map $x=(x(1), \ldots, x(m))$, we denote by the same notation $x$ the path map $(x(1), \ldots, x(m), 0, \ldots, 0)$. We shall identify two polygons or path maps if they are same as figures.

Under such identification, we define the direct sum of path maps. Let $x=(x(1), \ldots, x(h)), y=(y(1), \ldots, y(m))$ and $z=$ $(z(1), \ldots, z(n))$ be path maps. If $h=\max \{h, m, n\}$ and $x(i)=y(i)+$ $z(i)$ for every polygon $\{x(i), y(i), z(i)\}$, we say $x$ is the direct sum of $y$ and $z$, and we write $x=y \oplus z$.

REMARK 3. If we use the method of path model in [4], a polygon corresponds to a matrix algebra and a path map corresponds to a multi-matrix algebra. 
EXAMPLE. (1) The polygon $a_{6}(1)=\left(a_{1}(1) \rightarrow a_{6}(1)\right)$ and the path map $a_{6}=\left(a_{1}(1) \rightarrow a_{6}\right)$ are as follows in the case of either $\lambda \leq 1 / 4$ or $n \geq 6$ :
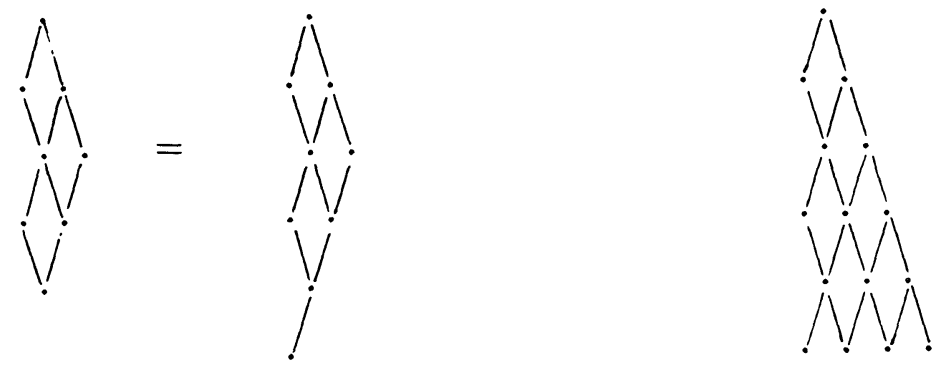

(2) Let $x \in \Xi_{7}, y \in \Xi_{6}$ and $Z \in \Xi_{6}$ be polygons, then $x=y \oplus z$ are as follows:

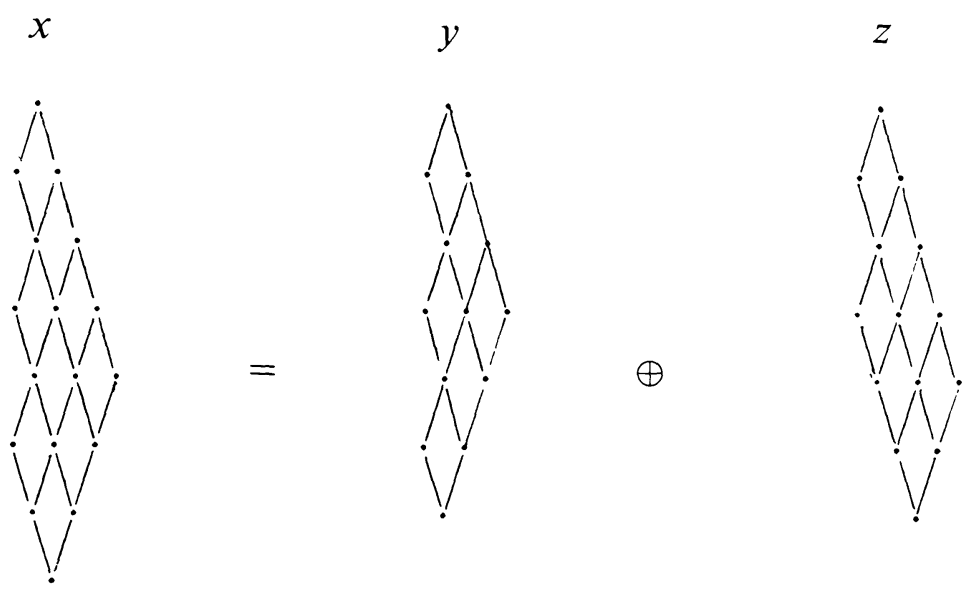

(3) Direct sum of path maps.
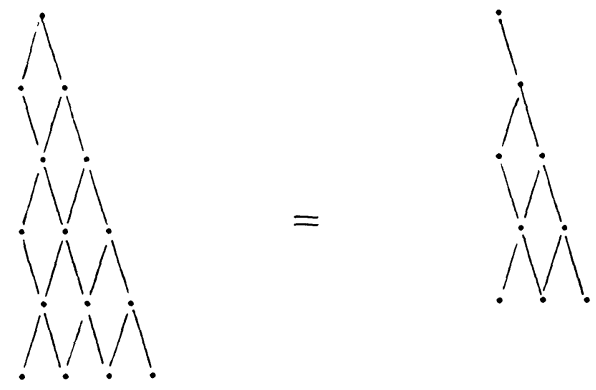

$\oplus$

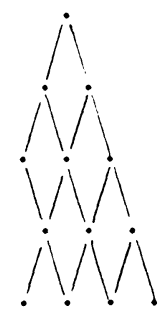


Now we discuss the inclusion matrix $\left[M_{q} \rightarrow M_{k}\right]$. It is obvious that the $(i, j)$-component of $\left[M_{q} \rightarrow M_{k}\right]$ means the cardinal number of $\left[a_{q}(i) \rightarrow a_{k}(j)\right]$. Hence the $i$ th row vector $x_{i}$ of $\left[M_{q} \rightarrow M_{k}\right]$ is considered as the path map $\left[a_{q}(i) \rightarrow a_{k}\right]$.

Under the identification of vectors and path maps, we define the polynomials $f_{i}(m)$ of path maps on $\Lambda$ by

$$
f_{i}(0)=a_{i}, \quad f_{i}(1)=a_{i+1} \quad \text { and } \quad f_{i}(m+1)=f_{i+1}(m) \ominus f_{i}(m-1) .
$$

Then for all positive integers $i$ and $m, f_{i}(2 m)$ (resp. $\left.f_{i}(2 m+1)\right)$ is a polynomial on path maps $\left\{a_{i+2 j} ; j=0,1,2, \ldots, m\right\}$ (resp. $\left\{a_{i+2 j+1}\right.$; $j=0,1,2, \ldots, m\}$ with positive integers as coefficients.

LEMMA 4. Let $x_{i}$ be the ith row vector of the inclusion matrix $\left[M_{q} \rightarrow\right.$ $\left.M_{k}\right]$, for a triplet $\{k, p, q\}$ in (3.1). Then, the path map $x_{i}$ is as follows for all $i\left(i=1,2, \ldots, d_{q}\right)$;

$$
x_{i}= \begin{cases}f_{p}(2 i-2) & \text { if } q \text { is even } \\ f_{p}(2 i-1) & \text { if } q \text { is odd }\end{cases}
$$

under the identification for vectors that $(y(1), \ldots, y(m), 0, \ldots, 0)=$ $(y(1), \ldots, y(m))$ for $y(j) \neq 0(j=1, \ldots, m)$.

Proof. Since the path map $x_{1}$ is $\left(a_{q}(1) \rightarrow a_{k}\right)$, it is clear by the shape of graph $\Lambda$ that

$$
x_{1}= \begin{cases}a_{p+1}=f_{p}(1) & \text { if } q \text { is odd } \\ a_{p}=f_{p}(0) & \text { if } q \text { is even }\end{cases}
$$

Suppose the statements are true for all $j \leq i$. As a path map, we have

$$
x_{i+1}=\left[a_{q}(i+1) \rightarrow a_{k}\right]= \begin{cases}{\left[a_{2 i}(i+1) \rightarrow a_{p+2 i}\right]} & \text { if } q \text { is even, } \\ {\left[a_{2 i+1}(i+1) \rightarrow a_{p+2 i+1}\right]} & \text { if } q \text { is odd, }\end{cases}
$$

by sliding up the line combining $a_{q}(1)$ and $a_{q}(i+1)$ as possible. Then the assumptions of the induction means that

$$
\left[a_{2(i-1)}(i) \rightarrow a_{p+2 i-2}\right]=f_{p}(2 i-2)
$$

and

$$
\left[a_{2(i-1)+1}(i) \rightarrow a_{p+2(i-1)+1}\right]=f_{p}(2 i-1) .
$$

Since

$$
\left[a_{2 i}(i) \rightarrow a_{p+2 i}\right] \oplus\left[a_{2 i}(i+1) \rightarrow a_{p+2 i}\right]=\left[a_{2 i-1}(i) \rightarrow a_{p+2 i}\right],
$$


we have

$$
\begin{aligned}
& {\left[a_{2 i}(i+1) \rightarrow a_{p+2 i}\right]=\left[a_{2 i-1}(i) \rightarrow a_{p+2 i}\right] \ominus\left[a_{2 i}(i) \rightarrow a_{p+2 i}\right]} \\
& \quad=\left[a_{2(i-1)+1}(i) \rightarrow a_{p+1+2(i-1)}\right] \ominus\left[a_{2(i-1)}(i) \rightarrow a_{p+2(i-1)}\right] \\
& \quad=f_{p+1}(2 i-1) \ominus f_{p}(2 i-2)=f_{p}(2 i) .
\end{aligned}
$$

On the other hand,

$$
\begin{gathered}
{\left[a_{2 i+1}(i) \rightarrow a_{p+2 i+1}\right] \oplus\left[a_{2 i+1}(i+1) \rightarrow a_{p+2 i+1}\right]} \\
=\left[a_{2 i}(i+1) \rightarrow a_{p+2 i+1}\right] .
\end{gathered}
$$

Hence

$$
\begin{aligned}
& {\left[a_{2 i+1}(i+1) \rightarrow a_{p+2 i+1}\right]} \\
& \quad=\left[a_{2 i}(i+1) \rightarrow a_{p+1+2 i}\right] \ominus\left[a_{2(i-1)+1}(i) \rightarrow a_{p+2(i-1)+1}\right] \\
& \quad=f_{p+1}(2 i) \ominus f_{P}(2 i-1)=f_{p}(2 i+1) .
\end{aligned}
$$

Thus $x_{i+1}=f_{p}(2 i)$ if $q$ is even and $x_{i+1}=f_{p}(2(i+1)-1)$ if $q$ is odd.

4. Bratteli diagram for $\left(N_{k}\right)_{k}$. Let $\left(N_{k}\right)$ be the sequence in (2.3). Let $N_{k}(+)=\left\{e_{i} \in N_{k} ; j \geq 1\right\}^{\prime \prime}$ and $N_{k}(-)=\left\{e_{j} \in N_{k} ; j \leq-1\right\}^{\prime \prime}$. Then $N_{k}$ is generated by the commuting pair $N_{k}(+)$ and $N_{K}(-)$. For a triplet $\{k, p, q\}$ in (3.1), $N_{k}(+)$ is isomorphic to $M_{q}$ and $N_{k}(-)$ is isomorphic to $M_{p}$. Two dimension vectors and weight vectors of a finite dimensional von Neumann algebra are respectively conjugate by an inner automorphism. We may take a dimension vector $b_{k}$ of $N_{k}$ and the weight vector $u_{k}$ for the restriction of the trace $\operatorname{tr}$ of $M$ to $N_{k}$ as

$$
b_{k}=\left(a_{p}(1) a_{q}, a_{p}(2) a_{q}, \ldots, a_{p}\left(d_{p}\right) a_{q}\right)
$$

and

$$
{ }^{t} u_{k}=\left(t_{p}(1)^{t} w_{q}, t_{p}(2)^{t} w_{q}, \ldots, t_{p}\left(d_{p}\right)^{t} w_{q}\right),
$$

where ${ }^{t} y$ denotes the transposed vector of the vector $y$. Since we obtained the inclusion matrices for $\left(M_{k}\right)$ in (3.1),

$$
\left[N_{k} \rightarrow N_{k+1}\right]= \begin{cases}I_{p} \otimes\left[M_{p} \rightarrow M_{p+1}\right] & \text { if } k \text { is odd, } \\ {\left[M_{p} \rightarrow M_{p+1}\right] \otimes I_{q}} & \text { if } k \text { is even, }\end{cases}
$$

where $I_{k}$ denotes the $d_{k}$ by $d_{k}$ identity matrix. It is easy to check that [ $N_{k} \rightarrow N_{k+1}$ ] satisfies the property (e) for $b_{k}$ and $u_{k}$. The Bratteli diagram for $\left(N_{k}\right)$ comes from the diagram for $\left(M_{k}\right)$ following after the above information.

In the case $\lambda=(1 / 4) \sec ^{2}(\pi / n+2)$ for some $n(n=1,2, \ldots)$, the diagram for $N_{1}=N_{2} \subset N_{3} \subset \cdots \subset N_{2 n}$ has the same form as in the 
case $\lambda \leq 1 / 4$, the diagram for $N_{2 n+4 i-2} \subset N_{2 n+4 i-1}$ (resp. $N_{2 n+4 i-1} \subset$ $\left.N_{2 n+4 i}\right)$ is similar to one for $N_{2 n-2} \subset N_{2 n-1}$ (resp. $N_{2 n-1} \subset N_{2 n}$ ) and the diagram for $N_{2 n+4 i} \subset N_{2 n+4 i+1}$ (resp. $N_{2 n+4 i+1} \subset N_{2 n+4 i+2}$ ) has the reverse form of order changed one for $N_{2 n-1} \subset N_{2 n}$ (resp. $\left.N_{2 n-2} \subset N_{2 n}\right)$.

5. Inclusion matrix of $N_{k}$ in $M_{k}$. Let $\{k, p, q\}$ be a triplet in (3.1). Let $x_{i}(j)$ be the $(i, j)$-component of $\left[M_{q} \rightarrow M_{k}\right]$ and $x_{i}$ the $i$ th column vector of $\left[M_{q} \rightarrow M_{k}\right]$. Here we consider $x(i, j)$ and $x_{i}$ as a polygon and a path map in $\Xi_{p}$. By Lemma 4 , the polygon $x_{i}(j)$ can be decomposed into the direct sum of polygons $\left\{a_{p+j}(i) ; j=0,1, \ldots, i=1,2, \ldots, d_{p}\right\}$. Then we define the matrix $\left[a_{p} \rightarrow x_{i}\right]=[h(j, k)]$ such that $h(j, k)$ is the number that $a_{p}(j)$ is contained in $x_{i}(k)$. We call the matrix $\left[a_{p} \rightarrow x_{i}\right]$ the inclusion matrix of the path map $a_{p}$ in the path map $x_{i}$.

REMARK 5. Let $x, y$ and $z$ be path maps on $\Lambda$ such that $[x \rightarrow y]$ and $[x \rightarrow z]$ are defined. Then, by the definition of the direct sum of path maps and the inclusion matrix for path maps, the matrix $[x \rightarrow(y \oplus z)]$ is defined and

$$
[x \rightarrow(y \oplus z)]=[x \rightarrow y]+[x \rightarrow z] .
$$

By this property and Lemma 4 , the inclusion matrix $\left[a_{p} \rightarrow x_{i}\right]$ of the path map $a_{p}$ in the path map $x_{i}$ is defined from the inclusion matrices $\left[M_{p} \rightarrow M_{r}\right](r \geq p)$ by the natural method.

LeMma 6. Let $\lambda=(1 / 4) \sec ^{2}(\pi / n+2)$ and $p \geq n-1$.

(1) If $n$ is odd and $p$ is even, then

$$
\begin{aligned}
& {\left[a_{p} \rightarrow f_{p}(m)\right](i, j)} \\
& \quad= \begin{cases}1, & -\left[\frac{m}{2}\right] \leq i-j \leq\left[\frac{m+1}{2}\right],\left[\frac{m}{2}\right]+2 \leq i+j \leq 2\left[\frac{n}{2}\right]-\left[\frac{m-1}{2}\right], \\
0, & \text { otherwise. }\end{cases}
\end{aligned}
$$

If $n$ is odd and $p$ is odd, then

$$
\begin{aligned}
& {\left[a_{p} \rightarrow f_{p}(m)\right](i, j)} \\
& \quad= \begin{cases}1, & -\left[\frac{m+1}{2}\right] \leq i-j \leq\left[\frac{m}{2}\right], 1+\left[\frac{m-1}{2}\right] \leq i+j \leq 2\left[\frac{n}{2}\right]-\left[\frac{m}{2}\right], \\
0, & \text { otherwise. }\end{cases}
\end{aligned}
$$

(2) If $n$ is even and $p$ is odd, then

$$
\begin{aligned}
& {\left[a_{p} \rightarrow f_{p}(m)\right](i, j)} \\
& \quad= \begin{cases}1, & -\left[\frac{m+1}{2}\right] \leq i-j \leq\left[\frac{m}{2}\right], 1+\left[\frac{m+1}{2}\right] \leq i+j \leq 2\left[\frac{n}{2}\right]-\left[\frac{m}{2}\right], \\
0, & \text { otherwise. }\end{cases}
\end{aligned}
$$


If $n$ is even and $p$ is even, then

$$
\begin{aligned}
& {\left[a_{p} \rightarrow f_{p}(m)\right](i, j)} \\
& \quad= \begin{cases}1, & -\left[\frac{m}{2}\right] \leq i-j \leq\left[\frac{m+1}{2}\right],\left[\frac{m}{2}\right]+2 \leq i+j \leq 2\left[\frac{n}{2}\right]-\left[\frac{m+1}{2}\right], \\
0, & \text { otherwise. }\end{cases}
\end{aligned}
$$

Proof. It is sufficient to prove the statement for $p=n-1$ and $p=n$, because $f_{p}(m)$ is the polynomial on $\left\{a_{p+j} ; j=[m / 2], j\right.$ is odd (resp. even) $\}$ if $m$ is odd (resp. even) and $\left[a_{p} \rightarrow a_{p+j}\right]=\left[a_{p+2} \rightarrow a_{p+2+j}\right]$ for all $p \geq n-1$ and $j$. Since $f_{p}(1)=a_{p+1}$, it is clear that $\left[a_{p} \rightarrow f_{p}(1)\right]$ satisfies the conditions for all $n$ and $p$. For a given $n$, assume that the statements hold for $p=n-1, n$ and $m=1,2, \ldots, k$. Then we can give a proof of the statements for $p=n-1, n$ and $m=k+1$ by the relation;

$$
\left[a_{p} \rightarrow f_{p}(k+1)\right]=\left[a_{p} \rightarrow a_{p+1}\right]\left[a_{p+1} \rightarrow f_{p+1}(k)\right]-\left[a_{p} \rightarrow f_{p}(k-1)\right]
$$

and

$$
\left[a_{n+1} \rightarrow f_{n+1}(k)\right]=\left[a_{n-1} \rightarrow f_{n-1}(k)\right]
$$

LEMMA 7. Let $\lambda=(1 / 4) \sec ^{2}(\pi / n+2)$ and $x_{i}$ the ith column vector of $\left[M_{q} \rightarrow M_{k}\right]$. Assume $q \geq n$.

(1) If $n$ is odd, then $\left[a_{p} \rightarrow x_{i}\right]$ is a $(1+[n / 2])$ square matrix with the following form:

(5.1) If $p=q$ is an odd number, then

$$
\left[a_{p} \rightarrow x_{i}\right](j, r)= \begin{cases}1, & 1-i \leq r-j \leq i<j+r \leq n+2-i, \\ 0, & \text { otherwise. }\end{cases}
$$

(5.2) If $p+1=q$ is even, then

$$
\left[a_{p} \rightarrow x_{i}\right](j, r)= \begin{cases}1, & |r-j|<i \leq j+r \leq n+2-i, \\ 0, & \text { otherwise. }\end{cases}
$$

(5.3) If $p=q$ is even, then

$$
\left[a_{p} \rightarrow x_{i}\right](j, r)= \begin{cases}1, & |r-j|<i<j+r \leq n+3-i, \\ 0, & \text { otherwise. }\end{cases}
$$

(5.4) If $p+1=q$ is odd, then

$$
\left[a_{p} \rightarrow x_{i}\right](j, r)= \begin{cases}1, & -i \leq r-j<i<j+r \leq n+2-i, \\ 0, & \text { otherwise. }\end{cases}
$$

(2) Let $n$ be even. 
(6.1) If $p=q$ is odd, then $\left[a_{p} \rightarrow x_{i}\right]$ is an $n / 2$ by $1+(n / 2)$ matrix with

$$
\left[a_{p} \rightarrow x_{i}\right](j, r)= \begin{cases}1, & 1-i \leq r-j \leq i<j+r \leq n+2-i, \\ 0, & \text { otherwise. }\end{cases}
$$

(6.2) If $p+1=q$ is even, then $\left[a_{p} \rightarrow x_{i}\right]$ is an $n / 2$ square matrix with

$$
\left[a_{p} \rightarrow x_{i}\right](j, r)= \begin{cases}1, & |r-j|<i \leq j+r \leq n+2-i, \\ 0, & \text { otherwise. }\end{cases}
$$

(6.3) If $p=q$ is even, then $\left[a_{p} \rightarrow x_{i}\right]$ is a $1+(n / 2)$ square matrix with

$$
\left[a_{p} \rightarrow x_{i}\right](j, r)= \begin{cases}1, & |r-j|<i<j+r \leq n+3-i, \\ 0, & \text { otherwise }\end{cases}
$$

(6.4) If $p+1=q$ is odd, then $\left[a_{p} \rightarrow x_{i}\right]$ is a $1+(n / 2)$ by $n / 2$ matrix with

$$
\left[a_{p} \rightarrow x_{i}\right](j, r)= \begin{cases}1, & -i \leq r-j<i<j+r \leq n+2-i \\ 0, & \text { otherwise. }\end{cases}
$$

Proof. Let $n$ be odd. Then $d_{j}=d_{n-1}$ for all $j \geq n-1$. Since $d_{n-1}=[n / 2]+1,\left[M_{q} \rightarrow M_{k}\right]$ is a $1+[n / 2]$ square matrix. It means that $a_{j}(j \geq n-1)$ and each $x_{i}$ are path maps consisting of $1+[n / 2]$ polygons in $\Xi_{p+1}$. Similarly, if $n$ is even, then $a_{j}$ is a path map with $[n / 2]$ (resp. $[n / 2]+1)$ polygons for odd (resp. even) $j \geq n-1$. Hence $x_{i}$ is a path map with $[n / 2]$ (resp. $\left.[n / 2]+1\right)$ polygons if $k$ is odd (resp. even). Therefore by Lemma 5 and Lemma 7, the statements hold.

LEMMA 8. For the weight vector $w_{k}$ of the restriction of $\operatorname{tr}$ to $M_{k}$, we have

$$
\left[a_{p} \rightarrow x_{i}\right] w_{k}=w_{q}(i) w_{p} \quad\left(i=1,2, \ldots, d_{q}\right) .
$$

Proof. We denote the matrix $\left[\left[a_{p} \rightarrow a_{p+i}\right], 0, \ldots, 0\right]$ by the same notation $\left[a_{p} \rightarrow a_{p+i}\right]$, where 0 is the column vector with all components 0 . Then by the Bratteli diagram for $\left(M_{k}\right)$, we have for all $i(i=0,1, \ldots)$

$$
\left[a_{p} \rightarrow a_{p+i}\right] w_{k}=\lambda^{n(i)} w_{p} \text { for } n(i)=\left[\frac{q}{2}\right]-\left[\frac{i}{2}\right] .
$$

Since $x_{i}$ is given by the polynomials on $\left\{a_{p+j} ; j=0,1, \ldots\right\}$ by Lemma 4 , we have the statement by Lemma $5,(3.2)$ and the relation between the polynomial $f_{j}$ 's and $P_{j}$ 's, because

$$
w_{k}(i)=\lambda^{p+1-i} P_{k-1-2 p+2 i}(\lambda)
$$


where $P_{j}$ is the polynomial defined in [3] by $P_{1}(x)=P_{2}(x)=1$ and $P_{n+1}(x)=P_{n}(x)-x P_{n-1}(x)$.

Let $G_{k}$ be the $d_{p} d_{q}$ by $d_{k}$ matrix, the $\left(d_{q}(j-1)+i\right)$ th row vector of which is the $j$ th row vector of the matrix $\left[a_{p} \rightarrow x_{i}\right]$, where $i=$ $1,2, \ldots, d_{q}, j=1,2, \ldots, d_{p}$. That is, the transposed matrix ${ }^{t} G_{k}$ of $G_{k}$ is as follows;

$$
\begin{array}{r}
{ }^{t} G_{k}=\left[G[1]_{1}, G[2]_{1}, \ldots, G\left[d_{q}\right]_{1}, G[1]_{2}, \ldots, G\left[d_{q}\right]_{2}, \ldots,\right. \\
\left.G[1]_{d_{p}}, \ldots, G\left[d_{q}\right]_{d_{p}}\right],
\end{array}
$$

where $G[i]_{j}$ is the transposed vector of the $j$ th row vector of $\left[a_{p} \rightarrow x_{i}\right]$.

LEMMA 9. The matrix $G_{k}$ satisfies the following.

$$
b_{k} G_{k}=a_{k}, \quad G_{k} w_{k}=u_{k} \quad \& \quad G_{k}\left[M_{k} \rightarrow M_{k+1}\right]=\left[N_{k} \rightarrow N_{k+1}\right] G_{k+1} \text {, }
$$

where $a_{k}, b_{k}$ are dimension vectors of $M_{k}, N_{k}$ and $W_{k}, u_{k}$ are weight vectors of $M_{k}, N_{k}$.

Proof. Since $a_{q}\left[M_{q} \rightarrow M_{k}\right]=a_{k}$, we have, by the relation (4.1),

$$
b_{k} G_{k}=\sum_{i} a_{q}(i) a_{p}\left[a_{p} \rightarrow x_{i}\right]=\sum_{i} a_{q}(i) x_{i}=a_{k},
$$

where $i$ runs over $\left\{1,2, \ldots, d_{q}\right\}$.

Lemma 6 implies that $G_{k} w_{k}=u_{k}$, combining the definition of $G_{k}$ and (4.2).

If $\lambda>1 / 4$ and $k \geq 2 n$, by Lemma 7 , we have $G_{k}\left[M_{k} \rightarrow M_{k+1}\right]=$ $\left[N_{k} \rightarrow N_{k+1}\right] G_{k+1}$. For another case, we need a similar lemma as Lemma 7. Below we do not need such cases. Hence we omit the proof of such cases.

Thus we can get a method of inclusion of $N_{k}$ in $M_{k}$. Hence we denote $G_{k}$ by $\left[N_{k} \rightarrow M_{k}\right]$.

6. Periodicity of $\left(N_{k}\right)_{k} \subset\left(M_{k}\right)_{k}$ in the case $\lambda>1 / 4$. In this section, we assume that $\lambda=(1 / 4) \sec ^{2} \pi /(n+2)$ for some $n(n=1,2, \ldots)$.

LEMma 10. The sequence $\left(M_{k}\right)$ is periodic with period 2 and the sequence $\left(N_{k}\right)$ is periodic with period 4 .

Proof. Combining the discussions in (2.5) in $\S 3$ with results in [2], we have that the sequence $\left(M_{k}\right)$ is periodic with period 2 . The fact implies that $\left(N_{k}\right)$ is periodic with period 4 , by the Bratteli diagram for $\left(N_{k}\right)$. 
LEMMA 11. Let $x_{i}$ (resp. $\left.y_{i}\right)$ be the ith row vector of $\left[M_{q} \rightarrow M_{k}\right]$ (resp. $\left.\left[M_{q+2} \rightarrow M_{k+4}\right]\right)$. If $q \geq n$, then

$$
\left[a_{p} \rightarrow x_{i}\right]=\left[a_{p+2} \rightarrow y_{i}\right] \quad\left(i=1,2, \ldots, d_{q}\right) .
$$

Proof. First we remark that both $\left[M_{q} \rightarrow M_{k}\right]$ and $\left[M_{q+2} \rightarrow M_{k+4}\right]$ are $d_{q}$ by $d_{k}$ matrices, because $\left(M_{k}\right)$ is periodic with period 2 and $\left[M_{q+2} \rightarrow M_{k+4}\right]=\left[M_{q} \rightarrow M_{k}\right]\left[M_{k} \rightarrow M_{k+2}\right]$. Since $p=[k / 2]$ and $q=k-p$, we have $p+2=[(k+4) / 2]$ and $q+2=k+4-(p+2)$, that is, $\{k+4, p+2, q+2\}$ satisfies (3.1). Hence $x_{i}=f_{p}(2 i-2)$ (resp. $\left.x_{i}=f_{p}(2 i-1)\right)$ if and only if $y_{i}=f_{p+2}(2 i-2)$ (resp. $\left.f_{p+2}(2 i-1)\right)$. By the definition, $f_{j}(2 m)$ (resp. $f_{j}(2 m+1)$ ) is a linear combination on $\left\{a_{j}, a_{j+2}, \ldots, a_{j+2 m}\right\}$ (resp. $\left\{a_{j+1}, a_{j+3}, \ldots, a_{j+2 m+1}\right\}$ ) with integer coefficients. Therefore, by Remark 5 , we have $\left[a_{p} \rightarrow x_{i}\right]=\left[a_{p+2} \rightarrow y_{i}\right]$, because $\left(M_{k}\right)$ is periodic with period 2 .

LEMMA 12. The sequence $\left(N_{k}\right) \subset\left(M_{k}\right)$ is periodic.

Proof. We already proved that both $\left(M_{k}\right)$ and $\left(N_{k}\right)$ are periodic with same period 4. Hence it is sufficient to prove that

$$
\left[N_{k} \rightarrow M_{k}\right]=\left[N_{k+4} \rightarrow M_{k+4}\right] \text { for } k \geq 2 n \text {. }
$$

By the form of the matrix $\left[N_{k} \rightarrow M_{k}\right]=G_{k}$, it is nothing else but Lemma 11. Thus $\left(N_{k}\right) \subset\left(M_{k}\right)$ is periodic.

\section{Proof of Theorem.}

LEMMA 13. If $\lambda=(1 / 4) \sec ^{2}(\pi / m)$ for some $m(m=3,4, \ldots)$, then

$$
[M: N]=(m / 4) \operatorname{cosec}^{2}(\pi / m) \text {. }
$$

Proof. The factors $M$ and $N$ are generated by the periodic sequences $\left(N_{k}\right) \subset\left(M_{k}\right)$ of finite dimensional algebras. Hence, by [6, Theorem 1.5], for the weight vectors $w_{k}$ and $u_{k}$ of the restriction tr to $M_{k}$ and $N_{k}$, we have that $[M: N]=\left\|u_{k}\right\|_{2}^{2} /\left\|w_{k}\right\|_{2}^{2}$ for a large enough $k$. By (4.2),

$$
\left\|u_{k}\right\|_{2}^{2}=\left\|w_{p}\right\|_{2}^{2}\left\|w_{q}\right\|_{2}^{2} \text { for a }\{k, p, q\} \text { in (3.1). }
$$

Put $n=m-2$. Then we have

$$
[M: N]=\left\|u_{k}\right\|_{2}^{2} /\left\|w_{k}\right\|_{2}^{2} \text { for all } k \geq n-1 .
$$

Since $\left\|w_{k}\right\|_{2}^{2} /\left\|w_{k+2}\right\|_{2}^{2}=1 / \lambda$ for all $k \geq n-1$,

$$
[M: N]=\left\|w_{n-1}\right\|_{2}^{4} /\left\|w_{2(n-1)}\right\|_{2}^{2}=\left\|w_{n-1}\right\|_{2}^{2} / \lambda^{n-1} .
$$


By the discussion in 3,

$$
\begin{aligned}
\left\|w_{n-1}\right\|_{2}^{2}= & \sum_{j} \lambda^{2 j} P_{n-2 \jmath}(\lambda)^{2} \\
& \text { where } j \text { runs over }\left\{0,1, \ldots,\left[\frac{n-1}{2}\right]\right\} .
\end{aligned}
$$

On the other hand, by [3],

$$
P_{k}\left((1 / 4) \sec ^{2} \theta\right)=\sin k \theta / 2^{k-1} \cos ^{k-1} \theta \sin \theta \text { for all } k \text { and } \theta .
$$

Hence

$$
\begin{aligned}
{[M: N] } & =\frac{\sum_{j} \sin ^{2}(n-2 j) \pi /(n+2)}{\sin ^{2}(\pi /(n+2))} \\
& =\frac{\sum_{j}\{2-\exp (2(n-2 j) /(n+2)) \pi i-\exp (2(2 j-n) /(n+2)) \pi i\}}{4 \sin ^{2}(\pi /(n+2))} \\
& =((n+2) / 4) \operatorname{cosec}^{2}(\pi /(n+2))=(m / 4) \operatorname{cosec}^{2}(\pi / m),
\end{aligned}
$$

because $\sum_{j=1}^{k} \exp ((j / k) 2 \pi i)=0$, for all integer $k$.

REMARK. 14 (1) If $m=3$ or 4 , then $[M: N]=[P: Q]$ for the subfactor $Q=\left\{e_{i} ; i=2,3, \ldots\right\}^{\prime \prime}$ of the factor $P=\left\{e_{i} ; i=1,2, \ldots\right\}^{\prime \prime}$. That is, $[M: N]=1$ if $m=3$ and $[M: N]=2$ if $m=4$.

(2) If $m \geq 5$, then $[M: N] \neq[P: Q]$. If $m=5$, then $[M: N]<4$. Hence there is an integer $k(k \geq 3)$ such that $[M: N]=4 \cos ^{2}(\pi / k)$. $\mathrm{H}$. Choda gets the number $k$, that is $k=10$. (Here the author thanks $\mathrm{H}$. Choda for helping her by computing a lot of indices $[M: N]$.) On the other hand, by the proof of Lemma 14,

$$
[M: N]=4 \cos ^{2}(\pi / 3)+4 \cos ^{2}(\pi / 5) \text {. }
$$

This implies the following equation (the equation is proved by an elementary method, which M. Fujii tells us);

$$
\cos ^{2}(\pi / 3)+\cos ^{2}(\pi / 5)=\cos ^{2}(\pi / 10) .
$$

The following lemma is an easy consequence of Skau's theorem ([7]). Here we shall denote another proof of it as an application of Lemma 7.

LEMMA 15. The relative commutant $N^{\prime} \cap M$ of $N$ in $M$ is trivial.

Proof. Since $[M: N]$ is finite, $N^{\prime} \cap M$ is finite dimensional. Let $c$ be the dimension vector of $N^{\prime} \cap M$. Since $\left(M_{k}\right) \supset\left(N_{k}\right)$ is periodic, by [6, Theorem 1.7],

$$
\|c\|_{1} \leq \alpha=\min \left\{\left\|G[i]_{j}\right\|_{1} ; k \geq 2 n, i=1,2, \ldots, d_{q}, j=1,2, \ldots, d_{p}\right\},
$$


where $G[i]_{j}$ is the vector in $\S 5$. By Lemma 8 , there are many $\{i, j\}$ 's such that ${ }^{t} G[i]_{j}=(1,0, \ldots, 0)$. It implies $\alpha=1$. Hence $N^{\prime} \cap M$ is 1-dimensional, so that $N^{\prime} \cap M=\mathrm{C} 1$.

8. A generalization. Fix a positive integer $n$. Let

$$
L=\left\{\ldots, e_{-n-1}, e_{-n}, e_{1}, e_{2}, e_{3}, \ldots\right\}^{\prime \prime} .
$$

In the case $n=1, L=N$. It is clear that $L$ is a subfactor of $M$, for all $n$. Also, $L$ is a subfactor of $N$ and $[N: L]=4 \cos ^{2}(\pi / m)$. Hence

$$
[M: L]=(m / 4) \operatorname{cosec}^{2}(\pi / m)\left\{4 \cos ^{2}(\pi / m)\right\}^{n-1} \text {. }
$$

Let

$$
L_{1}=L_{2}=\mathbf{C}_{1}, \quad L_{2 i-1}=L_{2 i}=\left\{e_{i} ; i=1,2, \ldots, n-1\right\}^{\prime \prime} \quad \text { if } i \leq n
$$

and

$$
L_{2 i+1}=\left\{L_{2 i}, e_{i}\right\}^{\prime \prime}, \quad L_{2 i+2}=\left\{e_{-i}, L_{2 i+1}\right\}^{\prime \prime} \quad \text { if } i \geq n .
$$

The sequence $\left(L_{k}\right)$ is periodic with period 4 and generates $L$. By a similar method as for $\left(N_{k}\right) \subset\left(M_{k}\right)$, we get the inclusion matrix $\left[L_{k} \rightarrow M_{k}\right.$ ]. For a triplet $\{k, p, q\}$ in (3.1), we consider the matrix $\left[a_{p-(n-1)} \rightarrow x_{i}\right]$ for a large $k$, where $x_{i}$ is the same as in $\S 3$, that is the $i$ th row vector of $\left[M_{q} \rightarrow M_{k}\right]$. Then $\left(N_{k}\right) \subset\left(M_{k}\right)$ is periodic. Let $h$ be the dimension vector of $L^{\prime} \cap M$.

If $q$ is even, then $x_{1}=a_{p}$; hence $\left[a_{p-(n-1)} \rightarrow x_{1}\right]=\left[a_{p-(n-1)} \rightarrow a_{p}\right]$.

If $n=2$, we have $N^{\prime} \cap M=\mathrm{C} 1$, by the form of $\left[a_{k} \rightarrow a_{k+1}\right]$ for an odd $k$.

If $n \geq 3,\left\{e_{-n+2}, e_{-n+3}, \ldots, e_{-1}\right\}^{\prime \prime}$ is contained in $L^{\prime} \cap M$ and isomorphic to $M_{n-1}$. Hence we have

$$
L^{\prime} \cap M=\left\{e_{-n+2}, e_{-n+3}, \ldots, e_{-1}\right\}^{\prime \prime} .
$$

\section{REFERENCES}

[1] O. Bratteli, Inductive limits of finite dimensional $C^{*}$-algebras, Trans. Amer. Math. Soc., 171 (1972), 195-234.

[2] F. Goodman, P. de la Harpe, and V. Jones, Coxeter-Dynkin diagrams and towers of algebras, preprint, I.H.E.S.

[3] V. Jones, Index for subfactors, Invent. Math. 72 (1983), 1-25.

[4] F. Murray, and J. von Neumann, On rings of operators, II, Trans. Amer. Math. Soc., 41 (1937), 208-248.

[5] M. Pimsner, and S. Popa, Entropy and index for subfactors, Ann. Sci. Ecole Norm. Sup., 19 (1986), 57-106.

[6] H. Wenzl, Representations of Hecke algebras and subfactors, Thesis, University of Pennsylvania. 
[7] F. Goodman, P. de la Harpe and V. Jones, Commuting squares, subfactors, and the derived tower, preprint.

Received October 10, 1987 and in revised form July 4, 1988.

OSAKa KyoIKu UNIVERSITY

TenNoJi, Osaka 543, JAPAN 


\section{PACIFIC JOURNAL OF MATHEMATICS EDITORS}

\author{
V. S. VARADARAJAN \\ (Managing Editor) \\ University of California \\ Los Angeles, CA 90024-1555-05 \\ Herbert Clemens \\ University of Utah \\ Salt Lake City, UT 84112 \\ THOMAS ENRIGHT \\ University of California, San Diego \\ La Jolla, CA 92093
}

R. FINN

Stanford University

Stanford, CA 94305

Hermann FlaschKa

University of Arizona

Tucson, AZ 85721

VAughan F. R. Jones

University of California

Berkeley, CA 94720

SteVen KerCKHOFF

Stanford University

Stanford, CA 94305
ROBION KIRBY

University of California

Berkeley, CA 94720

C. C. Moore

University of California

Berkeley, CA 94720

HAROLD STARK

University of California, San Diego

La Jolla, CA 92093

\section{ASSOCIATE EDITORS}

R. ARENS

E. F. BECKENBACH

B. H. NeumanN

F. WOLF

K. YoshIDA (1906-1982)

\section{SUPPORTING INSTITUTIONS}

UNIVERSITY OF ARIZONA

UNIVERSITY OF BRITISH COLUMBIA

CALIFORNIA INSTITUTE OF TECHNOLOGY

UNIVERSITY OF CALIFORNIA

MONTANA STATE UNIVERSITY

UNIVERSITY OF NEVADA, RENO

NEW MEXICO STATE UNIVERSITY

OREGON STATE UNIVERSITY
UNIVERSITY OF OREGON

UNIVERSITY OF SOUTHERN CALIFORNIA

STANFORD UNIVERSITY

UNIVERSITY OF HAWAII

UNIVERSITY OF TOKYO

UNIVERSITY OF UTAH

WASHINGTON STATE UNIVERSITY

UNIVERSITY OF WASHINGTON 


\section{Pacific Journal of Mathematics}

Vol. 139, No. $1 \quad$ May, 1989

Marie Choda, Index for factors generated by Jones' two sided sequence of

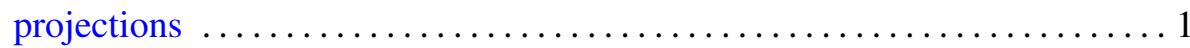

Bernadette Deshommes, Sur les zéros des fonctions symétriques complètes

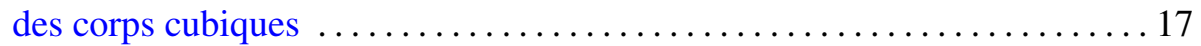

James K. Deveney and Joe Yanik, Nonrational fixed fields .............45

Mario Eudave-Muñoz, Prime knots obtained by band sums $\ldots \ldots \ldots \ldots \ldots 5$

Charles Dale Frohman, An unknotting lemma for systems of arcs in $F \times I \quad \ldots 59$

Alejandro Illanes, Spaces of Whitney maps ..................... 67

Konstantinos Karanikas, Wiener pairs of measure algebras . . . . . . . . 79

Ulrich Koschorke and Dale Rolfsen, Higher-dimensional link operations

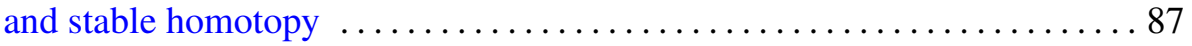

Wayne L. Neidhardt, Translation to and fro over Kac-Moody algebras . . . 107

Iain Raeburn, Allan M. Sinclair and Dana Peter Williams, Equivariant

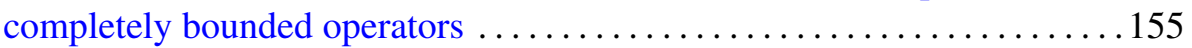

Katsuro Sakai and Raymond Y. T. Wong, On the space of Lipschitz homeomorphisms of a compact polyhedron 